\title{
Blood coagulation factor drives arthritis pathogenesis
}

Research now published in Blood reveals that the thrombin-activated coagulation transglutaminase factor XIII (fXIII) is an important factor in the pathogenesis of inflammatory arthritis, contributing to the disease through distinct mechanisms linked to local joint inflammation and altered osteoclast function.

"Elevated coagulation system activity, including fibrin deposition within affected joints, is a well-established pathological feature of patients with arthritis that is recapitulated in animal models of inflammatory joint disease," explains author Matthew Flick. Previous work by his group demonstrated that fibrinogen exacerbates collagen-induced arthritis (CIA) in mice, in part by binding the leukocyte integrin receptor $\alpha_{M} \beta_{2}$ and thus driving local inflammation. "The present report was an extension of these studies," continues Flick, "to determine whether fXIII, which is required to stabilize fibrin matrices at sites of injury, also promotes arthritis pathogenesis."

The researchers first showed that genetic elimination of fXIII limits the incidence and severity of CIA in mice. Joint pathology, including cartilage degradation and bone erosion, was diminished in $\mathrm{XXIIa^{-/- }}$ mice relative to wild-type $\left(\mathrm{XIIIa}^{+/+}\right)$littermates. Notably, $\mathrm{XIIIa}^{-/-}$ mice with CIA had a qualitatively distinct pattern of fibrin deposition in the knees associated with fewer inflammatory cells in the joint space, as well as reduced local expression of proinflammatory cytokines after induction of CIA.

"These data support the concept that fXIII can promote arthritis through mechanisms linked to proinflammatory activity that seem to be fibrin-dependent," reports Flick. "Unexpectedly, we also provide data to suggest that fXIII promotes arthritis through mechanisms linked to osteoclastogenesis that seem to be

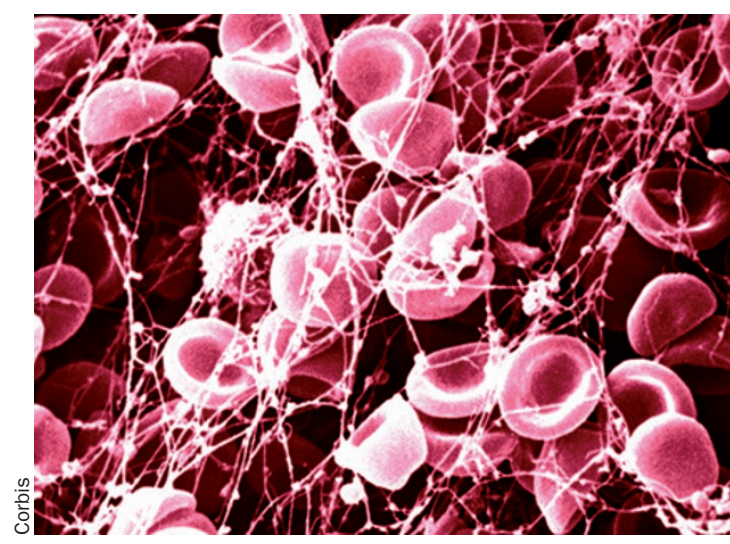

fibrin-independent." Unlike fibrinogendeficient mice, $f \mathrm{XIII}^{-/-}$mice had reduced osteoclast numbers and activity, which were linked to deficient receptor activator of nuclear factor $\mathrm{\kappa B}$ ligand-induced osteoclast formation.

Consistent with the genetic studies, pharmacological inhibition of transglutaminase activity diminished the development of CIA and osteoclastogenesis in wild-type mice, suggesting the therapeutic potential of targeting fXIII for inflammatory arthritis.

\section{Sarah Onuora}

Original article Raghu, H. et al. Transglutaminase factor XIII promotes arthritis through mechanisms linked to inflammation and bone erosion. Blood doi:10.1182/ blood-2014-08-594754 